\title{
The application of dual-layer spectral detector computed tomography in solitary pulmonary nodule identification
}

\author{
Qingyun Wen ${ }^{1}$, Yong Yue ${ }^{1}$, Jin Shang ${ }^{1}$, Xiaomei $\mathrm{Lu}^{2}$, Lu Gao ${ }^{3}$, Yang Hou ${ }^{1}$ \\ ${ }^{1}$ Department of Radiology, Shengjing Hospital of China Medical University, Shenyang, China; ${ }^{2}$ CT Clinical Science, Philips Healthcare, Shenyang, \\ China; ${ }^{3}$ Department of Radiology, Liaoning Cancer Hospital, Shenyang, China
}

Correspondence to: Yang Hou. Department of Radiology, Shengjing Hospital of China Medical University, No. 36, Sanhao Street, Shenyang 110004, China. Email: houyang1973@163.com.

Background: Differentiating between malignant solitary pulmonary nodules (SPNs) and other lung diseases remains a substantial challenge. The latest generation of dual-energy computed tomography (CT), which realizes dual-energy technology at the detector level, has clinical potential for distinguishing lung cancer from other benign SPNs. This study aimed to evaluate the performance of dual-layer spectral detector CT (SDCT) for the differentiation of SPNs.

Methods: Spectral images of 135 SPNs confirmed by pathology were retrospectively analyzed in both the arterial phase (AP) and the venous phase (VP). Patients were classified into two groups [the malignant group $(n=93)$ and the benign group $(n=42)$, with the malignant group further divided into small cell lung cancer (SCLC, n=30) and non-small cell lung cancer (NSCLC, n=63) subtypes. The slope of the spectral Hounsfield Unit (HU) curve $\left(\lambda_{\mathrm{HU}}\right)$, normalized iodine concentration (NIC), CT values of $40 \mathrm{keV}$ monochromatic images $\left(\mathrm{CT}_{40 \mathrm{keV}}\right)$, and normalized arterial enhancement fraction (NAEF) in contrast-enhanced images were calculated and compared between the benign and malignant groups, as well as between the SCLC and NSCLC subgroups. ROC curve analysis was performed to assess the diagnostic performance of the above parameters. Seventy cases were randomly selected and independently measured by two radiologists, and intraclass correlation coefficient (ICC) and Bland-Altman analyses were performed to calculate the reliability of the measurements.

Results: Except for NAEF $(\mathrm{P}=0.23)$, the values of the parameters were higher in the malignant group than in the benign group (all $\mathrm{P}<0.05)$. NIC, $\lambda_{\mathrm{HU}}$, and $\mathrm{CT}_{40 \mathrm{keV}}$ performed better in the $\mathrm{VP}\left(\mathrm{NIC}_{\mathrm{VP}}, \lambda_{\mathrm{VPHU}}\right.$, and $\left.\mathrm{CT}_{\mathrm{VP} 40 \mathrm{keV}}\right)(\mathrm{P}<0.001)$, with an area under the ROC curve $(\mathrm{AUC})$ of $0.93,0.89$, and 0.89 respectively. With respective cutoffs of $0.31,1.83$, and $141.00 \mathrm{HU}$, the accuracy of $\mathrm{NIC}_{\mathrm{VP}}, \lambda_{\mathrm{VPHU}}$, and $\mathrm{CT}_{\mathrm{VP} 40 \mathrm{keV}}$ was $91.11 \%$, $85.19 \%$, and $88.15 \%$, respectively. In the subgroup differentiating NSCLC and SCLC, the diagnostic performances of $\mathrm{NIC}_{\mathrm{AP}}(\mathrm{AUC}=0.89)$ were greater than other parameters. $\mathrm{NIC}_{\mathrm{AP}}$ had an accuracy of $86.02 \%$ when the cutoff was 0.14. ICC and Bland-Altman analyses indicated that the measurement of SDCT has great reproducibility.

Conclusions: Quantitative measures from SDCT can help to differentiate benign from malignant SPNs and may help with the further subclassification of malignant cancer into SCLC and NSCLC.

Keywords: Qualitative research; radiography; dual-energy scanned projection; solitary pulmonary nodules; tomography; X-ray computed tomography

Submitted Jan 02, 2020. Accepted for publication Sep 18, 2020.

doi: 10.21037/qims-20-2

View this article at: http://dx.doi.org/10.21037/qims-20-2 


\section{Introduction}

Low-dose computed tomography (LDCT) has recently been recommend for the screening of lung cancer, which has led to a significant improvement in the detection rate of solitary pulmonary nodules (SPNs). Early diagnosis and treatment of malignant SPNs are of utmost importance for prolonging survival and improving quality of life for patients. However, the evaluation of SPNs is still challenging $(1,2)$. The diagnosis of SPNs with conventional CT is mainly based on their morphological features, and a significant overlap in enhanced CT values poses a challenge in differentiating between benign and malignant lesions $(3,4)$. A multicenter study showed that the sensitivity of conventional contrast-enhanced CT for detecting benign and malignant pulmonary nodules was $96 \%$; however, the specificity was only $68 \%$ (5).

Recent investigations using source-based dual-energy CT techniques ( $\mathrm{kVp}$-switching and dual-source) have explored quantitative ways to evaluate pulmonary nodules through the assessment of various parameters (4,6-9). In a study describing the differentiation of SPNs using $\mathrm{kVp}$-switching energy CT, Zhang et al. concluded that the NIC, $\lambda_{\mathrm{HU}}$, and net CT number enhancement on $70 \mathrm{keV}$ images were higher in the benign group than in the malignant group $(\mathrm{P}<0.001)$ (4). However, using the same technology, Chen et al. found no significant differences in iodine concentration (IC) and $\lambda_{\mathrm{HU}}$ between benign and malignant solid SPNs $(\mathrm{P}>0.05)$ (7). Dual-source technology with iodine-based images has been shown to assist in identifying invasive and non-invasive lung cancer $(6,8)$ and in distinguishing lung squamous cell carcinoma from adenocarcinoma (9). Therefore, differences among dual-energy CT equipment and study samples can impact the findings and conclusions of studies.

The latest dual-layer spectral detector CT (SDCT) acquires homologous, in-phase, and temporally synchronized photons from a single source, thereby enabling energysensitive projection data to be obtained simultaneously. The system applies anti-correlated noise suppression and an iterative algorithm, thus reducing image noise and improving image quality (10-12). Another advantage that SDCT has over other types of dual-energy CT is that it can generate all spectral datasets without the need to pre-set dual-energy protocols, thereby efficient clinical workflow (13-16). However, there are still no studies focusing on the application of iodine quantitative parameters from SDCT for the differentiation of benign and malignant SPNs or the pathological classification of malignant nodules.

Hence, using pathology as the gold standard, we aimed to investigate the usefulness of iodine-based quantitative parameters from SDCT for differentiating between benign and malignant SPNs. We also sought to further subclassify small cell lung cancer (SCLC) and non-small cell lung cancer (NSCLC) in the diagnosis of SPNs in order to facilitate improved clinical decision-making regarding timely and optimal therapy for patients.

\section{Methods}

\section{Patients}

One hundred and fifty-one patients who underwent SDCTenhanced scans at the hospital between December 2016 and July 2019 and were found to have SPNs were initially considered for inclusion in the study. The inclusion criteria were as follows: (I) all SPNs detected on SDCT were pathologically confirmed (gold standard) and untreated; (II) the diameter of the SPN was $\leq 30 \mathrm{~mm}$ in the lung window. The exclusion criteria were as follows: (I) nodules appearing as pure ground-glass opacity (pGGO) or as subsolid nodules and measuring $<8 \mathrm{~mm}$ in diameter in the lung window that could not be measured in the mediastinal window; (II) patients who received chemoradiotherapy prior to the SDCT scan; (III) patients with $>1$ nodule.

Of the 151 patients initially considered, 7 were excluded because they received chemoradiotherapy before the SDCT scan, and 9 were excluded because the SPN manifested as a ground-glass nodule (GGN). Ultimately, data from 135 patients were collected and analyzed (Figure 1). Of these, 59 cases were confirmed by postoperative pathology, 47 cases by percutaneous biopsy, and 29 cases by biopsy performed during the bronchoscopy. Detailed information of the patient characteristics and pathologic results is shown in Table 1. This retrospective diagnostic study was approved by ethics committee of Shengjing Hospital of China Medical University (2016PS016J).

\section{CT examinations}

Chest scans were performed in both the arterial phase (AP) and the venous phase (VP) on a dual-layer spectral detector CT (IQon Spectral CT, Philips Healthcare, Cleveland, OH, USA). All patients were scanned in the supine position, with the scanning range from the thoracic inlet to the costophrenic angle to ensure coverage of all lung tissues. 


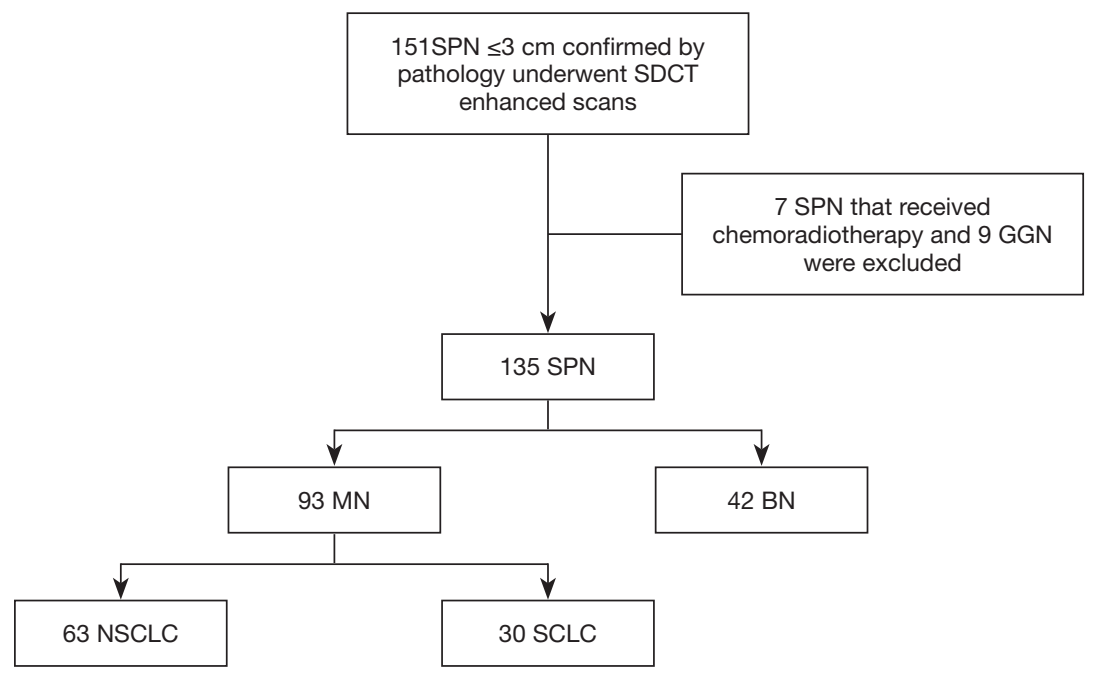

Figure 1 Flow chart of patient selection. SDCT, dual-layer spectral detector CT; SPN, solitary pulmonary nodule; GGN, ground-glass nodule; BN, benign pulmonary nodule; MN, malignant pulmonary nodule; SCLC, small cell lung cancer; NSCLC, non-small cell lung cancer.

Table 1 Patient characteristics and pathologic results

\begin{tabular}{lc}
\hline Variable & Value $^{\dagger}$ \\
\hline Total PN & 135 \\
Age (years) & $61.52 \pm 6.60$ \\
Sex & \\
Male & $74(54.81)$ \\
Female & $61(45.19)$ \\
Pathology & \\
Malignant SPN & $93(68.89)$ \\
Adenocarcinoma & $38(28.15)$ \\
Squamous cell carcinoma & $25(18.52)$ \\
$\quad$ SCLC & $30(22.22)$ \\
Benign SPN & $42(31.11)$ \\
Inflammatory granuloma & $17(12.59)$ \\
Inflammatory myofibroblastoma & $4(2.96)$ \\
Tuberculosis & $21(15.56)$ \\
Effective dose (mSv) & $10.61 \pm 1.33$ \\
\hline${ }^{\dagger}$ Data are presented as the mean \pm SD or $\mathrm{n}(\%)$. The effective \\
dose includes the radiation dose received in the arterial and \\
venous phases. SPN, solitary pulmonary nodule; SCLC, small
\end{tabular}

The following scanning parameters were used: tube voltage: $120 \mathrm{kV}$; automatic tube current modulation with the Dose Right Index (DRI) set to 22 (this value was chosen to provide a balance between image quality and radiation dose); rotation speed: 0.33 s/rot; helical pitch: 0.671 ; detector collimation: $64 \times 0.625 \mathrm{~mm}$; image reconstruction matrix: $512 \times 512$. Two reconstruction kernels: Standard (B) and Y-Detail (YB) respectively for mediastinal window and lung window.

After a native chest scan, $80 \mathrm{~mL}$ of contrast agent (Iodixanol, $270 \mathrm{mg} / \mathrm{mL}$, GE Healthcare, Ireland) was injected with a power injector (Ulrich REF XD 2051) via the cubital vein, at a flow rate of $2.5 \mathrm{~mL} / \mathrm{s}$. Arterial and venous images were acquired at 25 and $60 \mathrm{~s}$ after the injection onset, respectively. The patients were trained in breath-holding before the examination to reduce the chance of motion artifacts during the scan and also to provide consistency between the two scans.

All images were reconstructed as Spectral Base Image (SBI) datasets, with the spectral reconstruction level set to 3, a reconstructed slice thickness of $1 \mathrm{~mm}$, and an increment of $1 \mathrm{~mm}$. Images reconstructed with the Standard kernel (B) were reviewed in a mediastinal window with a window width of $350 \mathrm{HU}$ and a level of $60 \mathrm{HU}$, and those reconstructed with Y-Detail (YB) were reviewed in the lung window with 
a window width of $-1,200 \mathrm{HU}$ at a level of $-600 \mathrm{HU}$.

\section{Image analysis}

The images were analyzed using a proprietary image workstation (IntelliSpace Portal 6.5, Philips Healthcare). Virtual monochromatic images (VMIs) at $40 \mathrm{keV}$ and $80 \mathrm{keV}$ and iodine density maps were used. Three regions of interest (ROIs) were manually outlined at the center of nodules in the middle of the homogeneous tissue. The copy-and-paste function was used to ensure that the size and position of the ROIs were the same between reconstructions obtained from the $\mathrm{AP}$ and $\mathrm{VP}$ scans. To verify the reliability and consistency of the measurement, 70 cases were randomly selected in a blinded manner and measured independently by two radiologists with 13 years and 10 years of experience in thoracic imaging, respectively. The Intraclass correlation coefficient (ICC) between the two radiologists was calculated. The final results of all cases were measured by the radiologist with 13 years' experience.

The following parameters were used in our study: (I) CT value (HU) from $40 \mathrm{keV}$ reconstructions $\left(\mathrm{CT}_{40 \mathrm{keV}}\right)$; (II) the slope of spectral HU curve $\left(\lambda_{\mathrm{HU}}\right)$ : CT values of $\mathrm{VMI}$ at $40 \mathrm{keV}\left(\mathrm{CT}_{40 \mathrm{keV}}\right)$ and $80 \mathrm{keV}\left(\mathrm{CT}_{80 \mathrm{keV}}\right)$ were selected and $\lambda_{\mathrm{HU}}$ $=\mid \mathrm{CT}_{40 \mathrm{keV}}-\mathrm{CT}_{80 \mathrm{keV}} \mathrm{I} /(80-40)$ (the changes in CT attenuation in this range were prominent in contrast to the higher energy levels, where the spectral curve exhibited smaller changes); (III) normalized iodine concentration (NIC): the iodine concentration (IC) values of the SPN ( $\mathrm{IC}_{\mathrm{SPN}}$ ) and of the thoracic aorta $\left(\mathrm{IC}_{\text {aorta }}\right)$ were measured from the same iodinebased slice and $\mathrm{NIC}=\mathrm{IC}_{\mathrm{SPN}} / \mathrm{IC}_{\text {aorta }}$ (IV) normalized arterial enhancement fraction (NAEF) was defined as the NIC in the AP divided by the NIC in the VP. The values of $\mathrm{CT}_{40 \mathrm{keV}}, \lambda_{\mathrm{HU}}$, and NIC were measured from both the AP and VP scans.

\section{Statistical analysis}

SPSS 22.0.0 (IBM, Armonk, NY, USA) and MedCalc 15.2.2 (Ostend, Belgium) were used for all statistical analyses. Continuous variables were expressed as means \pm standard deviations or medians and interquartile ranges. The twosample $\mathrm{T}$ test or Mann-Whitney U-test was used to compare the differences of spectral parameters between the benign and malignant groups as well as between the SCLC and NSCLC subgroups. Receiver operating characteristic (ROC) curves were used to compare the differences among quantitative parameters. The Youden index was calculated to determine the parameter thresholds for SPN differentiation. The sensitivity, specificity, accuracy, positive predictive value (PPV), and negative predictive value (NPV) of $\mathrm{CT}_{40 \mathrm{keV}}, \lambda_{\mathrm{HU}}, \mathrm{NIC}$, and NAEF were determined to assess their diagnostic performances. ICC and BlandAltman analyses were performed with the data of 70 patients to quantify the reliability and reproducibility of the measurement. Statistical significance was defined as $\mathrm{P} \leq 0.05$.

\section{Results}

\section{Difference of iodine-related parameters between the benign and malignant groups}

The values of $\mathrm{CT}_{40 \mathrm{ke}}, \lambda_{\mathrm{HU}}$, and NIC were all higher in the malignant group than in the benign group (all $\mathrm{P}<0.05$; $\mathrm{NAEF}, \mathrm{P}=0.23$ ) (Figures 2 and 3). The optimal thresholds and diagnostic efficacy of the spectral CT parameters are shown in Table 2. The ROC curves showed that $\mathrm{NIC}_{\mathrm{VP}}$, $\lambda_{\mathrm{VPHU}}$, and $\mathrm{CT}_{\mathrm{VP} 40 \mathrm{keV}}$ had higher diagnostic capability, with $\mathrm{NIC}_{\mathrm{VP}}$ having the highest diagnostic performance, with an area under the curve (AUC) of 0.93 (Figure 4). However, there were no significant differences found in the diagnostic performances of $\mathrm{NIC}_{\mathrm{VP}}$ and the other three parameters mentioned above $(\mathrm{P}>0.05)$.

With the cutoff value for $\mathrm{NIC}_{\mathrm{VP}}$ set at 0.31 , there were 5 malignant nodules $(5 / 93,5.38 \%)$ wrongly identified as negative and 7 benign nodules (7/42, 16.67\%) wrongly identified as positive.

\section{Difference of iodine-related parameters between the SCLC and NSCLC groups}

The values of $\mathrm{CT}_{40 \mathrm{keV}}, \mathrm{NIC}$, and $\lambda_{\mathrm{HU}}$ were significantly higher in the NSCLC group than in the SCLC group $(\mathrm{P}<0.05)$ (Table 3, Figure 5). With an AUC of 0.89 , $\mathrm{NIC}_{\mathrm{AP}}$ showed a best diagnostic performance; however, its performance did not differ significantly from those of $\mathrm{NIC}_{\mathrm{VP}}, \lambda_{\mathrm{APHU}}, \lambda_{\mathrm{VPHU}}$, and $\mathrm{CT}_{\mathrm{AP} 40 \mathrm{keV}}(\mathrm{P}>0.05)$ (Table 4). The ROC curves of the parameters in the SCLC and NSCLC groups are compared in Figure 6.

With the cutoff of NIC set at 0.14 to differentiate SCLC and NSCLC in the AP, 4 cases $(4 / 63,6.35 \%)$ of NSCLC were misdiagnosed, and 9 cases $(9 / 30,30 \%)$ in the SCLC group had false-positive results.

\section{Reliability of measurements}

The ICC values for $\mathrm{CT}_{\mathrm{AP} 40 \mathrm{ke}}, \mathrm{CT}_{\mathrm{VP} 40 \mathrm{keV}}, \lambda_{\mathrm{APHU}}, \lambda_{\mathrm{VPHU}}$, 

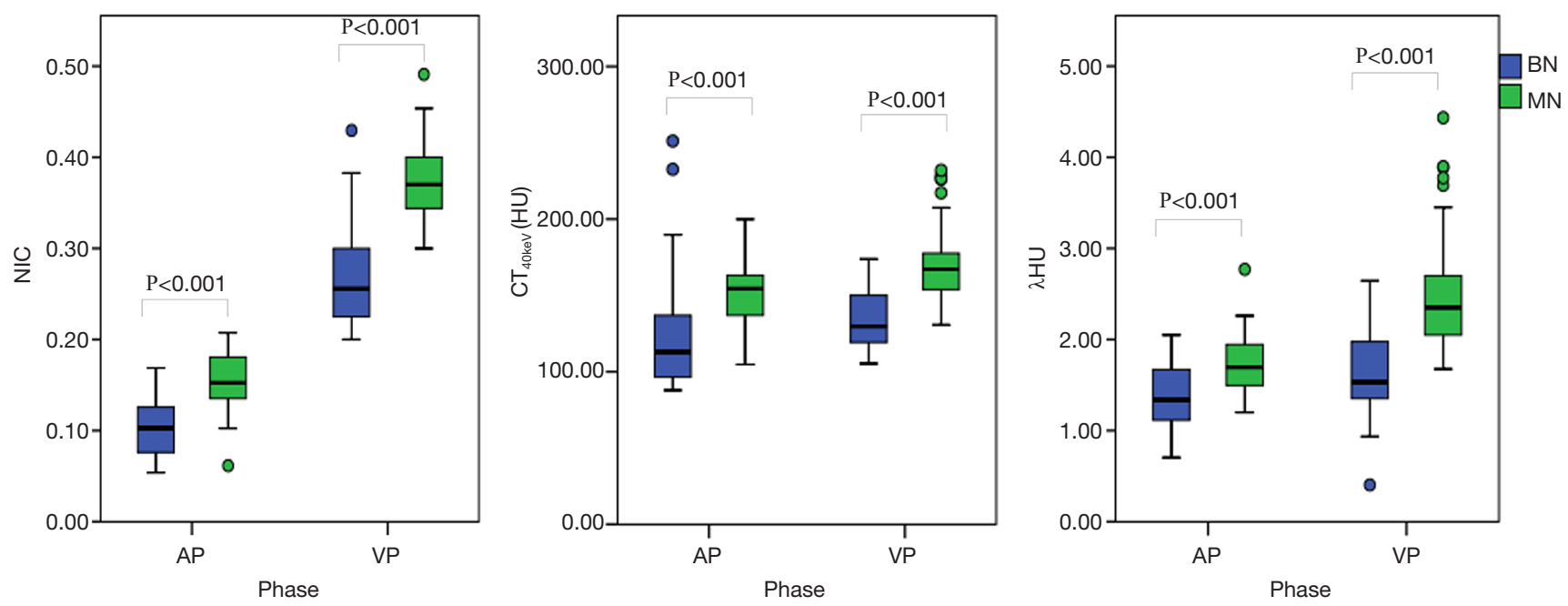

Figure 2 Boxplots showing a comparison between benign and malignant nodules as demonstrated by CT $\mathrm{T}_{40 \mathrm{ke}}, \lambda_{\mathrm{HU}}$, and NIC in both the AP and VP. All parameters had significantly higher values in the malignant group than in the benign group (all $\mathrm{P}<0.001)$. AP, arterial phase; VP, venous phase; $\mathrm{BN}$, benign pulmonary nodule; $\mathrm{MN}$, malignant pulmonary nodule; $\mathrm{CT}_{40 \mathrm{keV}}$, the $\mathrm{HU}$ value of $40 \mathrm{keV}$ monochromatic images at the measured location; $\lambda_{\mathrm{HU}}$, the slope of the spectral HU curve; NIC, the normalized iodine concentration.

$\mathrm{NIC}_{\mathrm{AP}}, \mathrm{NIC}_{\mathrm{VP}}$, and NAEF were $0.86,0.85,0.86,0.82,0.87$, 0.83 , and 0.84 , respectively (Table 5). The results of BlandAltman analysis are shown in Table 6.

\section{Discussion}

The results of this study showed that the iodine-related parameters of $\mathrm{CT}_{40 \mathrm{ke}}, \lambda_{\mathrm{HU}}$, and NIC from SDCT can be useful in differentiating benign and malignant SPNs. $\mathrm{NIC}_{\mathrm{VP}}$ showed the best diagnostic performance in this study, with an accuracy of $91.11 \%$. Furthermore, NIC $_{A P}$ showed an ability for further subclassification of SCLC and NSCLC. ICC and Bland-Altman analyses indicated that the measurement of SDCT has great reproducibility.

To our knowledge, this is the first study to identify the nature of SPNs using the iodine-related quantitative index from SDCT. According to our results, the value of NIC was greater in the malignant group than in the benign group, and $\mathrm{NIC}_{\mathrm{VP}}$ was superior in differentiating malignant from benign nodules. This result was similar to the findings of previous studies that demonstrated the sensitivity and specificity of $\mathrm{NIC}_{\mathrm{VP}}$ to be in the ranges of $93-93.8 \%$ and 85.7-95\%, respectively (4,17-19). Xiao et al. (18) focused on the value of $\mathrm{kVp}$-switching dual-energy CT in SPN diagnosis. They scanned 42 benign SPNs and 20 malignant SPNs and demonstrated that the value of NIC was higher in malignant nodules than benign nodules $(\mathrm{P}<0.05)$.
Furthermore, Zhang et al. (4) reported that NIC was a more valuable index than $\lambda_{\mathrm{HU}}$ and that CT numbers, especially in the VP, demonstrated a sensitivity and specificity of $93.8 \%$ and $85.7 \%$, respectively. Another study regarding the distribution difference of iodine concentration between malignant and benign SPNs found that the differences in NIC (dNIC) between the proximal and the distal regions in the VP were optimal in the characterization of SPNs, with a sensitivity and specificity of $93 \%$ and $95 \%$, respectively (17).

The reason why iodine-related parameters are of great value in identifying benign and malignant SPNs may relate to tumor vascularity. The iodine concentration directly reflects the blood supply within the lesion $(20,21)$. Compared with benign SPNs, malignant SPNs tend to grow more blood vessels in order to feed and support their own rapid growth, resulting in higher iodine-related values. The parameter of NIC, standardized by the iodine concentration of the aorta, minimizes the influence of hemodynamic factors among different individuals on the absolute enhancement of lesions, and consequently increases the comparability between different cases, thus making NIC more specific than other iodine-related indicators.

Additionally, some studies have shown that iodinerelated parameters in inflammatory benign lesions have higher values than those in malignant lesions $(22,23)$. The reason for the difference in the results observed in our study could be related to the cases enrolled. In our study, half of 

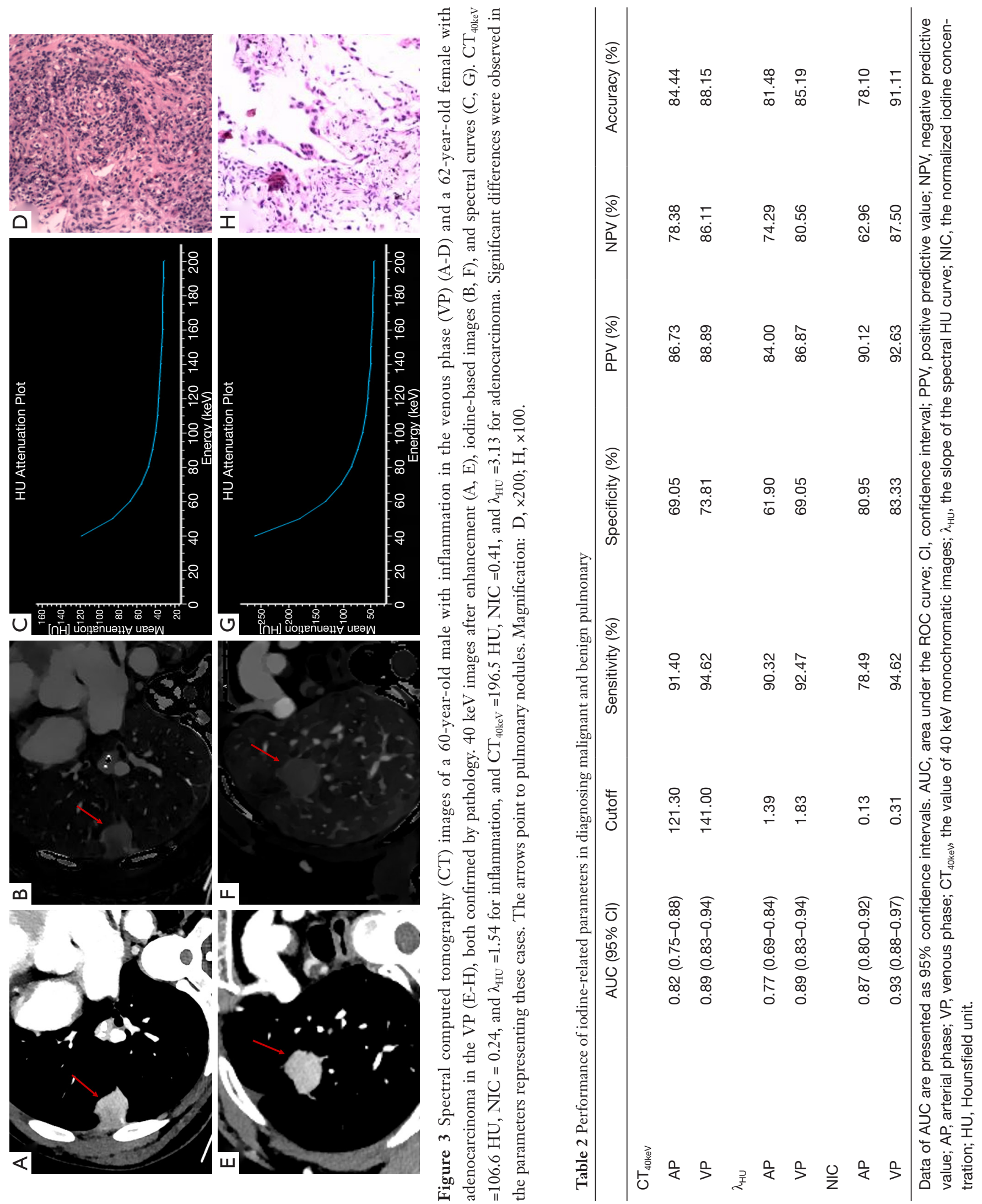


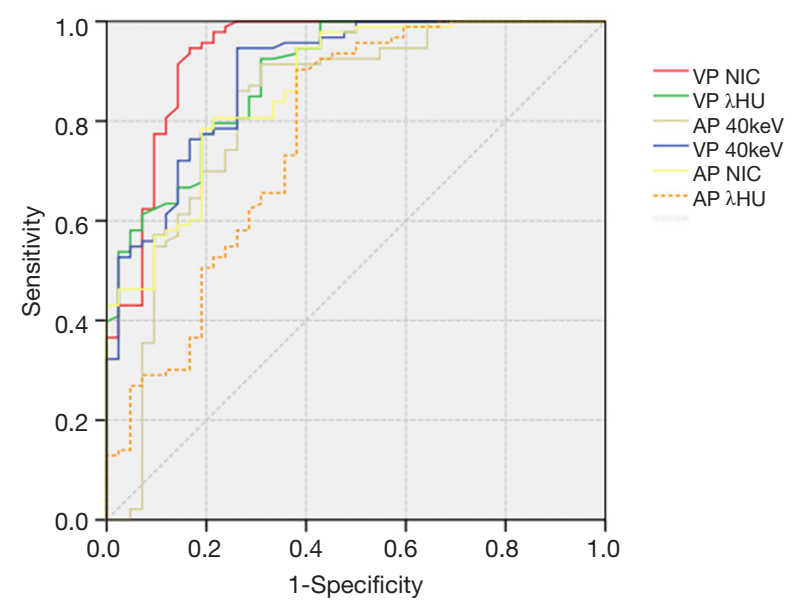

Figure 4 Receiver operating characteristic curves for distinguishing benign from malignant nodules using iodine-related quantitative parameters. The $\mathrm{AUC}$ of $\mathrm{NIC}_{\mathrm{VP}}\left(\mathrm{AUC}_{\mathrm{VPNIC}}\right)$ was 0.93 (95\% CI: 0.88-0.97), the AUC of $\mathrm{CT}_{\mathrm{VP} 40 \mathrm{keV}}$ was 0.89 (95\% CI: $\left.0.83-0.94\right)$, and the AUC of $\lambda_{\mathrm{VP} H U}$ was 0.89 (95\% CI: 0.83-0.94). AUC, area under the curve; NIC, normalized iodine concentration; VP, venous phase.

the benign SPNs were tuberculosis (21/42, 50\%), and the remainder comprised chronic inflammation (granuloma and myofibroblastoma), with no acute inflammation found. SPNs due to tuberculosis lack blood supply, and these nodules are mainly composed of a necrotic core surrounded by granulated or fibrous tissue. As such, these nodules with chronic inflammation exhibit reduced blood supply due to the destruction of blood vessels (24). However, nodules with acute inflammation included in other studies have capillaries that are stimulated by inflammatory factors and become dilated, thus making the values of spectral parameters in these lesions higher than those in malignant lesions.

According to our research, the use of VMIs of $40 \mathrm{keV}$ from SDCT may be an effective way to characterize SPN. Many studies on other types of dual-energy technologies have focused on $70 \mathrm{keV}$ images in disease diagnosis $(4,23,25)$. The reason for this may be that lower $\mathrm{keV}$ VMIs have increased image noise, which lowers the potential of these reconstructions for diagnosing disease $(26,27)$. However, with the detector-based approach of SDCT, the spatially and temporally matched high- and low-energy projection data facilitates the exploitation of anti-correlated noise suppression, leading to a reduction in noise in VMIs with lower $\mathrm{keV}$ and an improvement in
Table 3 Comparison of computed tomography iodine-related parameters between small cell lung cancer and non-small cell lung cancer

\begin{tabular}{|c|c|c|c|c|}
\hline & SCLC & NSCLC & $\mathrm{t}$ value & $P$ value \\
\hline \multicolumn{5}{|c|}{$\mathrm{CT}_{40 \mathrm{keV}}(\mathrm{HU})$} \\
\hline $\mathrm{AP}$ & $135.31 \pm 21.71$ & $149.58 \pm 22.41$ & 6.042 & $<0.001$ \\
\hline VP & $161.44 \pm 21.99$ & $170.46 \pm 19.13$ & 1.955 & 0.054 \\
\hline \multicolumn{5}{|l|}{$\lambda_{\mathrm{HU}}$} \\
\hline $\mathrm{AP}$ & $1.51 \pm 0.17$ & $1.79 \pm 0.23$ & 5.963 & $<0.001$ \\
\hline VP & $2.11 \pm 0.31$ & $2.61 \pm 0.28$ & 4.152 & $<0.001$ \\
\hline \multicolumn{5}{|l|}{$\mathrm{NIC}$} \\
\hline AP & $0.13 \pm 0.02$ & $0.17 \pm 0.02$ & 7.992 & $<0.001$ \\
\hline VP & $0.34 \pm 0.03$ & $0.39 \pm 0.04$ & 5.576 & $<0.001$ \\
\hline NAEF & $0.37 \pm 0.06$ & $0.44 \pm 0.08$ & 4.190 & $<0.001$ \\
\hline
\end{tabular}

$\mathrm{Cl}$, confidence interval; SCLC, small cell lung cancer; NSCLC, non-small cell lung cancer; $\mathrm{HU}$, Hounsfield unit; AP, artery phase; VP, venous phase; $\mathrm{CT}_{40 \mathrm{kev}}$, the value of $40 \mathrm{keV}$ monochromatic images; $\lambda_{\mathrm{HU}}$, the slope of spectral HU curve; NIC, the normalized iodine concentration; NAEF, the normalized arterial enhancement fraction.

image quality $(28,29)$. Our results showed that the accuracy of $\mathrm{CT}_{40 \mathrm{keV}}$ from AP and VP scans in differentiating malignant nodules from benign nodules was $84.44 \%$ and $88.15 \%$, respectively

Distinguishing SCLC and NSCLC is essential for accurate treatment and prognosis evaluation. In our study, the values of $\mathrm{CT}_{40 \mathrm{keV}}$, NIC, $\lambda_{\mathrm{HU}}$, and NAEF were significantly higher in the NSCLC group than in the SCLC group $(\mathrm{P}<0.05)$. Similarly, $\mathrm{Xu}$ et al. (30) evaluated iodine maps from dual-energy CT to distinguish SCLC from NSCLC, and confirmed that the IC values were markedly lower in SCLC compared with NSCLC. This result may be related to the differences in vascularization inside the nodules representing these two subgroups. The relatively large vessels of the microvascular beds in adenocarcinoma and squamous cell carcinoma are more abundant than those in SCLC (31). Furthermore, tumor necrosis occurs more frequently in $\operatorname{SCLC}(30,32)$. It is worth noting that in differentiating SCLC from NSCLC, the diagnostic performance of spectral parameters is higher in the AP than in the VP, which is different from the differentiation of benign and malignant pulmonary nodules. This may be related to the enhancement of the microvascular density in the AP, which is richer in NSCLC 

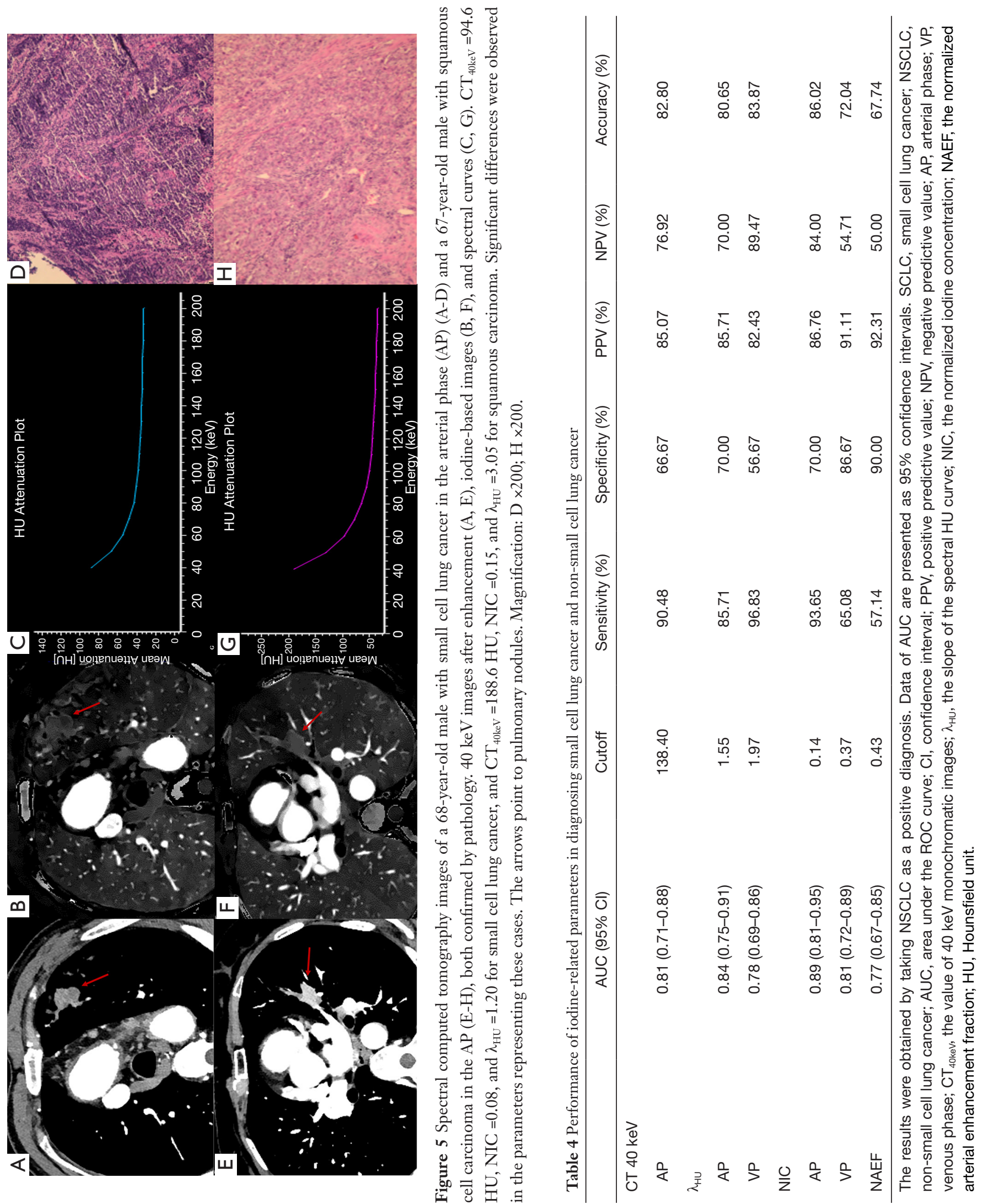


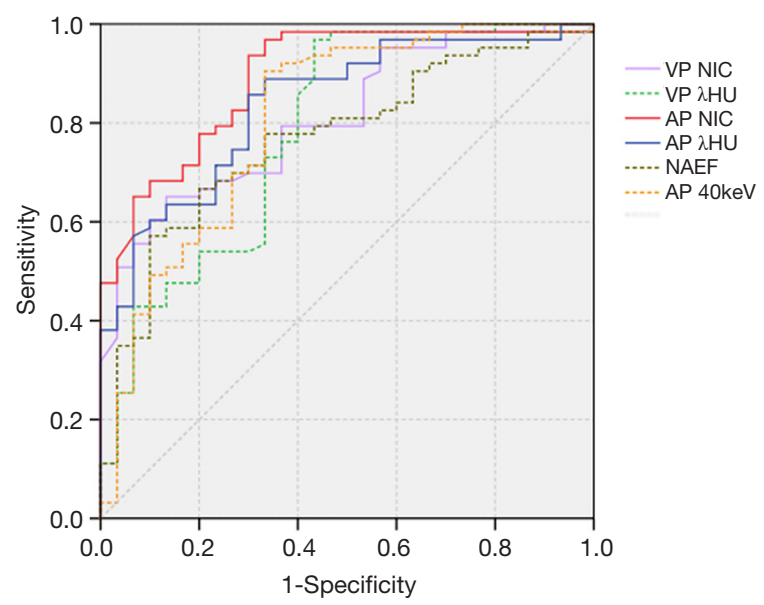

Figure 6 Receiver operating characteristic curves for distinguishing small cell lung cancer and non-small cell lung cancer using iodinerelated quantitative parameters. The area under the receiver operating characteristic curve of $\mathrm{NIC}_{\mathrm{AP}}$ was higher than those of the other parameters, with a value of 0.89 (95\% CI: 0.81-0.95). than in SCLC (33). Lacking the basement membrane, these abnormal vessels increase their permeability about blood flow to interstitial space, and the blockage of venous and lymphatic systems in malignant lesions makes iodine contrast agent accumulation in interstitial space. The difference in iodine concentration between SCLC and NSCLC decreases with time increase, which makes the AP more valuable than the VP in differentiating SCLC from NSCLC.

Using the cutoff of 0.31 in NIC $_{\mathrm{VP}}$ to differentiate malignant SPN from benign SPN, there were 5 malignant nodules ( 3 of which were SCLC) and 7 benign nodules (5 of which were inflammatory granulomas) that were misdiagnosed as SCLC. As mentioned earlier, NIC reflects the relative vascularity of pulmonary lesions, and a greater overlap exists between SCLC and benign SPNs than between NSCLC and benign SPNs.

Using the cutoff of 0.14 for $\mathrm{NIC}_{\mathrm{AP}}$ to differentiate between SCLC and NSCLC, there were 4 cases of NSCLC

Table 5 Inter-reader correlation with solitary pulmonary nodules differentiation

\begin{tabular}{|c|c|c|c|c|c|c|c|}
\hline & $\mathrm{CT}_{\mathrm{AP} 40 \mathrm{keV}}(\mathrm{HU})$ & $\mathrm{CT}_{\mathrm{VP} 40 \mathrm{keV}}(\mathrm{HU})$ & $\lambda_{\mathrm{APHU}}$ & $\lambda_{\mathrm{YPHU}}$ & $\mathrm{NIC}_{\mathrm{AP}}$ & $\mathrm{NIC}_{\mathrm{VP}}$ & NAEF \\
\hline
\end{tabular}

Table 6 Bland-Altman analysis performed by two radiologists

\begin{tabular}{lcccc}
\hline & $\mathrm{MD}(95 \% \mathrm{Cl})$ & $1.96 \mathrm{SD}$ & \multicolumn{2}{c}{ LOA } \\
\cline { 4 - 5 } & & & Lower (mean-1.96SD) & Upper (mean +1.96SD) \\
\hline $\mathrm{CT}_{\text {AP40keV }}$ & $-0.410(-3.6487,2.8278)$ & 26.619 & -27.029 & 26.208 \\
$\mathrm{CT}_{\text {VP40KVV }}$ & $-0.830(-4.2956,2.6356)$ & 28.489 & -29.317 & 27.658 \\
$\lambda_{\text {APHU }}$ & $-0.025(-0.065,0.016)$ & 0.334 & -0.359 & 0.310 \\
$\lambda_{\text {VPHU }}$ & $-0.057(-0.118,0.004)$ & 0.501 & -0.558 & 0.444 \\
$\mathrm{NIC}_{\text {AP }}$ & $-0.003(-0.007,0.002)$ & 0.037 & -0.039 & 0.034 \\
$\mathrm{NIC}_{\mathrm{VP}}$ & $-0.024(-0.033,-0.015)$ & 0.072 & -0.096 & 0.048 \\
NAEF & $-0.008(-0.018,0.003)$ & 0.088 & -0.096 & 0.080 \\
\hline
\end{tabular}

$\mathrm{MD}$, mean difference; SD, standard deviation; LOA, limit of agreement; $\mathrm{CT}_{\mathrm{AP} 40 \mathrm{keV}}$, the value of $40 \mathrm{keV}$ monochromatic images in the arterial phase; $\lambda_{\mathrm{APHU}}$, the slope of the spectral $\mathrm{HU}$ curve in the arterial phase; $\mathrm{NIC}_{\mathrm{AP}}$, the normalized iodine concentration in the arterial phase; $\mathrm{CT}_{\mathrm{VP} 40 \mathrm{keV}}$, the value of $40 \mathrm{keV}$ monochromatic images in the venous phase; $\lambda_{\mathrm{VPH}}$, the slope of the spectral HU curve in the venous phase; $\mathrm{NIC}_{\mathrm{VP}}$, the normalized iodine concentration in the venous phase; NAEF, normalized arterial enhancement fraction; HU, Hounsfield unit. 
(3 of which were squamous cell carcinomas) that were misdiagnosed as SCLC. This could be attributable to micro-vessel density. Squamous cell carcinoma, which is mainly formed by cancer cell stacking, manifests as a solid mass that can infringe on blood vessels, thus affecting the blood supply (34). Among the 4 cases of NSCLC that were misdiagnosed, 3 tumors were poorly differentiated and 1 was moderately to highly differentiated. Poorly differentiated carcinoma is characterized by more aggressive growth, with a lower micro-vessel density, leading to a lower value of NIC (35).

Previous investigations showed that the use of iodinerelated quantitative parameters from source-based dualenergy CT (fast $\mathrm{kVp}$ switching) improved the specificity for differentiating benign from malignant pulmonary nodules (17). However, the radiation dose for source-based dual-energy CT is higher than that for conventional CT, and an a priori decision is also required to select the scanning mode $(4,19)$. The use of SDCT, which applies anti-correlated noise suppression and iterative algorithm, obviates the need for scanning mode pre-setting with no additional radiation exposure, making it more convenient and efficient from a workflow perspective (28).

There are several limitations to this study. First, this was a single-center study with a limited number of lesions; thus, further investigations need to be performed in the future. Second, in this study the morphological characteristics of the nodules (such as tumor size, lobulation, and speculation), which are traditionally used to determine the potential of a nodule, were not evaluated. However, our study has shown that the quantitative parameters of iodine are excellent for distinguishing benign and malignant tumors (for instance, $\mathrm{NIC}_{\mathrm{VP}}$ has an accuracy of $91.11 \%$ ). In the future, we will further study the value of combining morphology with the iodine-based quantitative parameters of SDCT. Third, only the vendor-recommended spectral reconstruction (level 3) was used in this study, and future studies will need to be conducted to evaluate the impact of different iterative reconstruction levels.

In conclusion, iodine-related quantitative parameters obtained with SDCT imaging are useful in the characterization of pulmonary nodules. $\mathrm{NIC}_{\mathrm{VP}}, \lambda_{\mathrm{VPHU}}$, and $\mathrm{CT}_{\mathrm{VP} 40 \mathrm{keV}}$ are excellent for identifying benign and malignant SPNs, while $\mathrm{NIC}_{\mathrm{AP}}$ has shown the capability to further subclassify malignant SPNs into SCLC and NSCLC. Thus, SDCT is a promising modality that can provide noninvasive guidance for the accurate treatment and prognostic determination of lung cancer.

\section{Acknowledgments}

The authors would like to thank Mani Vembar and Zheng Jia, who helped us to prepare the text of this paper

Funding: This study was supported by the 345 Talent Project in Shengjing Hospital of China Medical University.

\section{Footnote}

Conflicts of Interest: All authors have completed the ICMJE uniform disclosure form (available at http://dx.doi. org/10.21037/qims-20-2). The authors have no conflicts of interest to declare.

Ethical Statement: This study was approved by the Ethics Committee of Shengjing Hospital of China Medical University (approval number, 2016PS016J). Written informed consent was obtained from all patients prior to study participation.

Open Access Statement: This is an Open Access article distributed in accordance with the Creative Commons Attribution-NonCommercial-NoDerivs 4.0 International License (CC BY-NC-ND 4.0), which permits the noncommercial replication and distribution of the article with the strict proviso that no changes or edits are made and the original work is properly cited (including links to both the formal publication through the relevant DOI and the license). See: https://creativecommons.org/licenses/by-nc-nd/4.0/.

\section{References}

1. Truong MT, Ko JP, Rossi SE, Rossi I, Viswanathan C, Bruzzi JF, Marom EM, Erasmus JJ. Update in the evaluation of the solitary pulmonary nodule. Radiographics 2014;34:1658-79.

2. Gould MK, Tang T, Liu IL, Lee J, Zheng C, Danforth KN, Kosco AE, Di Fiore JL, Suh DE. Recent Trends in the Identification of Incidental Pulmonary Nodules. Am J Respir Crit Care Med 2015;192:1208-14.

3. Erasmus JJ, Connolly JE, McAdams HP, Roggli VL. Solitary pulmonary nodules: Part I. Morphologic evaluation for differentiation of benign and malignant lesions. Radiographics 2000;20:43-58.

4. Zhang Y, Cheng J, Hua X, Yu M, Xu C, Zhang F, $\mathrm{Xu}$ J, Wu H. Can Spectral CT Imaging Improve the Differentiation between Malignant and Benign Solitary Pulmonary Nodules? PLoS One 2016;11:e0147537. 
5. Swensen SJ, Viggiano RW, Midthun DE, Müller NL, Sherrick A, Yamashita K, Naidich DP, Patz EF, Hartman TE, Muhm JR, Weaver AL. Lung nodule enhancement at CT: multicenter study. Radiology 2000;214:73-80.

6. Shimamoto H, Iwano S, Umakoshi H, Kawaguchi K, Naganawa S. Evaluation of locoregional invasiveness of small-sized non-small cell lung cancers by enhanced dual-energy computed tomography. Cancer Imaging 2016;16:18.

7. Chen ML, Li XT, Wei YY, Qi LP, Sun YS. Can spectral computed tomography imaging improve the differentiation between malignant and benign pulmonary lesions manifesting as solitary pure ground glass, mixed ground glass, and solid nodules? Thorac Cancer 2019;10:234-42.

8. Son JY, Lee HY, Kim JH, Han J, Jeong JY, Lee KS, Kwon OJ, Shim YM. Quantitative CT analysis of pulmonary ground-glass opacity nodules for distinguishing invasive adenocarcinoma from non-invasive or minimally invasive adenocarcinoma: the added value of using iodine mapping. Eur Radiol 2016;26:43-54.

9. Zhang Z, Zou H, Yuan A, Jiang F, Zhao B, Liu Y, Chen J, Zuo M, Gong L. A Single Enhanced Dual-Energy CT Scan May Distinguish Lung Squamous Cell Carcinoma From Adenocarcinoma During the Venous phase. Acad Radiol 2020;27:624-9.

10. Kim J, Lee KH, Kim J, Shin YJ, Lee KW. Improved repeatability of subsolid nodule measurement in low-dose lung screening with monoenergetic images: a phantom study. Quant Imaging Med Surg 2019;9:171-9.

11. Lu X, Lu Z, Yin J, Gao Y, Chen X, Guo Q. Effects of radiation dose levels and spectral iterative reconstruction levels on the accuracy of iodine quantification and virtual monochromatic CT numbers in dual-layer spectral detector CT: an iodine phantom study. Quant Imaging Med Surg 2019;9:188-200.

12. Ozguner O, Dhanantwari A, Halliburton S, Wen G, Utrup S, Jordan D. Objective image characterization of a spectral CT scanner with dual-layer detector. Phys Med Biol 2018;63:025027.

13. Forghani R, Mukherji SK. Advanced dual-energy CT applications for the evaluation of the soft tissues of the neck. Clin Radiol 2018;73:70-80.

14. Rassouli N, Etesami M, Dhanantwari A, Rajiah P. Detector-based spectral CT with a novel dual-layer technology: principles and applications. Insights Imaging 2017;8:589-98.
15. Hickethier T, Byrtus J, Hauger M, Iuga AI, Pahn G, Maintz D, Haneder S, Doerner J. Utilization of virtual mono-energetic images (MonoE) derived from a duallayer spectral detector CT (SDCT) for the assessment of abdominal arteries in venous contrast phase scans. Eur J Radiol 2018;99:28-33.

16. Hojjati M, Van Hedent S, Rassouli N, Tatsuoka C, Jordan D, Dhanantwari A, Rajiah P. Quality of routine diagnostic abdominal images generated from a novel detector-based spectral CT scanner: a technical report on a phantom and clinical study. Abdom Radiol (NY) 2017;42:2752-9.

17. Wu L, Cao G, Zhao L, Tang K, Lin J, Miao S, Lin T, Sun J, Zheng X. Spectral CT Analysis of Solitary Pulmonary Nodules for Differentiating Malignancy from Benignancy: The Value of Iodine Concentration Spatial Distribution Difference. Biomed Res Int 2018;2018:4830659.

18. Xiao H, Liu Y, Tan H, Liang P, Wang B, Su L, Wang S, Gao J. A pilot study using low-dose Spectral CT and ASIR (Adaptive Statistical Iterative Reconstruction) algorithm to diagnose solitary pulmonary nodules. BMC Med Imaging 2015;15:54.

19. Lin JZ, Zhang L, Zhang CY, Yang L, Lou HN, Wang ZG. Application of Gemstone Spectral Computed Tomography Imaging in the Characterization of Solitary Pulmonary Nodules: Preliminary Result. J Comput Assist Tomogr 2016;40:907-11.

20. Jiang T, Zhu AX, Sahani DV. Established and novel imaging biomarkers for assessing response to therapy in hepatocellular carcinoma. J Hepatol 2013;58:169-77.

21. Apfaltrer P, Meyer M, Meier C, Henzler T, Barraza JM, Jr., Dinter DJ, Hohenberger P, Schoepf UJ, Schoenberg SO, Fink C. Contrast-enhanced dual-energy CT of gastrointestinal stromal tumors: is iodine-related attenuation a potential indicator of tumor response? Invest Radiol 2012;47:65-70.

22. Yu Y, Wang X, Shi C, Hu S, Zhu H, Hu C. Spectral Computed Tomography Imaging in the Differential Diagnosis of Lung Cancer and Inflammatory Myofibroblastic Tumor. J Comput Assist Tomogr 2019;43:338-44.

23. Hou WS, Wu HW, Yin Y, Cheng JJ, Zhang Q, Xu JR. Differentiation of lung cancers from inflammatory masses with dual-energy spectral CT imaging. Acad Radiol 2015;22:337-44.

24. Zhang M, Kono M. Solitary pulmonary nodules: evaluation of blood flow patterns with dynamic CT. Radiology 1997;205:471-8. 
25. Rassouli N, Chalian H, Rajiah P, Dhanantwari A, Landeras L. Assessment of 70-keV virtual monoenergetic spectral images in abdominal CT imaging: A comparison study to conventional polychromatic $120-\mathrm{kV}$ images. Abdom Radiol (NY) 2017;42:2579-86.

26. Matsumoto K, Jinzaki M, Tanami Y, Ueno A, Kuribayashi S. Virtual monochromatic spectral imaging with fast kilovoltage switching: improved image quality as compared with that obtained with conventional $120-\mathrm{kVp}$ CT. Radiology 2011;259:257-62.

27. Apfaltrer P, Sudarski S, Schneider D, Nance JW Jr, Haubenreisser H, Fink C, Schoenberg SO, Henzler T. Value of monoenergetic low-kV dual energy CT datasets for improved image quality of CT pulmonary angiography. Eur J Radiol 2014;83:322-8.

28. Doerner J, Hauger M, Hickethier T, Byrtus J, Wybranski C, Grosse Hokamp N, Maintz D, Haneder S. Image quality evaluation of dual-layer spectral detector CT of the chest and comparison with conventional CT imaging. Eur J Radiol 2017;93:52-8.

29. Bae K, Jeon KN, Cho SB, Park SE, Moon JI, Baek HJ, Choi BH. Improved Opacification of a Suboptimally Enhanced Pulmonary Artery in Chest CT: Experience Using a Dual-Layer Detector Spectral CT. AJR Am J Roentgenol 2018;210:734-41.

30. Xu X, Sui X, Zhong W, Xu Y, Wang Z, Jiang J, Ge Y,

Cite this article as: Wen Q, Yue Y, Shang J, Lu X, Gao L, Hou Y. The application of dual-layer spectral detector computed tomography in solitary pulmonary nodule identification. Quant Imaging Med Surg 2021;11(2):521-532. doi: 10.21037/qims-20-2
Song L, Du Q, Wang X, Song W, Jin Z. Clinical utility of quantitative dual-energy CT iodine maps and CT morphological features in distinguishing small-cell from non-small-cell lung cancer. Clin Radiol 2019;74:268-77.

31. Zieliński KW, Kulig A, Zieliński J. Morphology of the microvascular bed in primary human carcinomas of lung. Part II. Morphometric investigations of microvascular bed of lung tumors. Pathol Res Pract 1984;178:369-77.

32. Travis WD. Update on small cell carcinoma and its differentiation from squamous cell carcinoma and other non-small cell carcinomas. Mod Pathol 2012;25 Suppl 1:S18-30.

33. Shi J, Schmid-Bindert G, Fink C, Sudarski S, Apfaltrer P, Pilz LR, Liu B, Haberland U, Klotz E, Zhou C, Schoenberg S, Henzler T. Dynamic volume perfusion CT in patients with lung cancer: baseline perfusion characteristics of different histological subtypes. Eur J Radiol 2013;82:e894-900.

34. Padera TP, Stoll BR, Tooredman JB, Capen D, di Tomaso E, Jain RK. Pathology: cancer cells compress intratumour vessels. Nature 2004;427:695.

35. Lin LY, Zhang Y, Suo ST, Zhang F, Cheng JJ, Wu HW. Correlation between dual-energy spectral CT imaging parameters and pathological grades of non-small cell lung cancer. Clin Radiol 2018;73:412.e1-412.e7. 